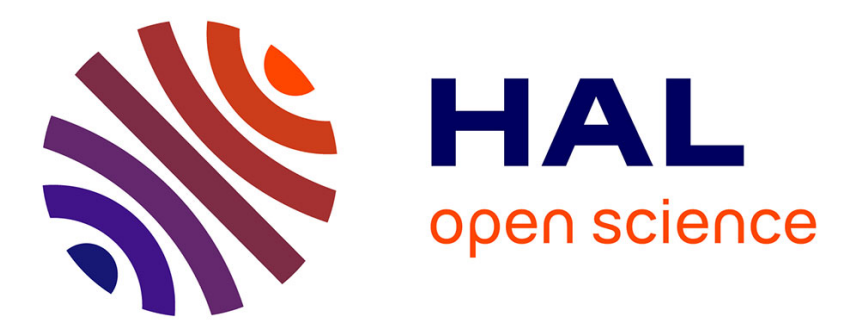

\title{
Computationally Efficient MR-FDPF and MR-FDTLM Methods for Multifrequency Simulations
}

Dmitry Umansky, Jean-Marie Gorce, Meiling Luo, Guillaume de La Roche, Guillaume Villemaud

\section{- To cite this version:}

Dmitry Umansky, Jean-Marie Gorce, Meiling Luo, Guillaume de La Roche, Guillaume Villemaud. Computationally Efficient MR-FDPF and MR-FDTLM Methods for Multifrequency Simulations. IEEE Transactions on Antennas and Propagation, 2012, 61 (3), pp.1309-1320. 10.1109/TAP.2012.2227920 . hal-00737356

\section{HAL Id: hal-00737356 https://hal.inria.fr/hal-00737356}

Submitted on 19 Dec 2013

HAL is a multi-disciplinary open access archive for the deposit and dissemination of scientific research documents, whether they are published or not. The documents may come from teaching and research institutions in France or abroad, or from public or private research centers.
L'archive ouverte pluridisciplinaire HAL, est destinée au dépôt et à la diffusion de documents scientifiques de niveau recherche, publiés ou non, émanant des établissements d'enseignement et de recherche français ou étrangers, des laboratoires publics ou privés. 


\title{
Computationally Efficient MR-FDPF and MR-FDTLM Methods for Multifrequency Simulations
}

\author{
Dmitry Umansky, Jean-Marie Gorce, Meiling Luo, Guillaume de la Roche, and Guillaume Villemaud
}

\begin{abstract}
We propose a modification of the multi-resolution frequency domain ParFlow (MR-FDPF) method that allows simulating radio propagation channels in a frequency range. The performance of the proposed MR-FDPF implementation has been analyzed based on different realistic propagation scenarios.

We also analyze the possibility of applying the multi-resolution frequency domain approach to the well-known transmission-line matrix method. The proposed multi-resolution frequency domain transmission-line matrix method provides a computationally efficient way of modeling radio wave propagation in threedimensional space at multiple frequencies.
\end{abstract}

Index Terms-ParFlow, TLM, wave propagation, indoor radio propagation, channel simulation, multiresolution, frequency domain, OFDM.

\section{INTRODUCTION}

$\mathbf{T}$ HE new generation of wireless communication systems, such as the IEEE 802.16 WiMAX and the 3GPP LongTerm Evolution (LTE), has recently reached the stage of actual deployment. Broadband wireless access (BWA) technologies constitute an indispensable part of these systems. The orthogonal frequency division multiplexing (OFDM) [1] is one of the key BWA techniques implemented in modern high data rate wireless communication systems.

Evaluation and optimization of OFDM systems performance calls for reliable models that adequately describe radio propagation channels over the whole system frequency bandwidth. The deterministic channel models based on the numerical simulation of Maxwell's equations [2], as well as the models based on the ray-optical methods [3], are known for their relatively high accuracy [4]. One of the main drawbacks of these modeling approaches is the large computational load. During the last decade, a great deal of research has been focused on finding an optimal trade-off between the computational complexity and the achievable accuracy of the deterministic channel modeling approaches. In [5], the multiresolution frequency domain ParFlow (MR-FDPF) method has been proposed. The method has proved to be efficient for modeling indoor and indoor-like environments. However,

D. Umansky, J.-M. Gorce, and Guillaume Villemaud are with the CITI, INSA-LYON, F-69621, Villeurbanne, France, and INRIA, Université de Lyon, F-69621, Villeurbanne, France, e-mail: (see http://www.citi.insalyon.fr/teams/swing/\#people).

M. Luo is with Ranplan Wireless Network Design Ltd, Suite 25, The Business Competitiveness Centre, Kimpton Road, LU2 0SX, Luton, UK.

G. de la Roche is with CWiND, University of Bedfordshire, Park Square Campus, Luton LU1 3JU, UK. similar to the majority of the frequency domain methods [6][8], the MR-FDPF algorithm has to be repeated for every frequency in the range of interest. Consequently, the computational load associated with the method quickly becomes excessively large as the number of frequencies increases. This is particularly the case for OFDM wireless communication systems, where data are transmitted in parallel over several sub-carriers. For example, LTE systems can operate with the bandwidths ranging from $1.4 \mathrm{MHz}$ and up to $20 \mathrm{MHz}$ with $15 \mathrm{kHz}$ subcarrier spacing [4].

The aim of this paper is twofold. Firstly, we develop a modification of the MR-FDPF method that allows computationally efficient simulations of the electromagnetic wave propagation in a range of frequencies. Then, utilizing the inherent similarities between the ParFlow method [9] and the well-known transmission line matrix (TLM) method [10], we show that the multi-resolution frequency domain (MR-FD) approach can be applied to the TLM method in order to efficiently model propagation media in a frequency range. In this regard, we can refer to the work presented in [11], where the authors proposed the transient frequency domain transmissionline matrix (TFDTLM) approach to model propagation media in a frequency range by running only one simulation. The TFDTLM achieves the computational savings by employing digital filters to model the electromagnetic wave propagation in the frequency dispersive media.

The rest of the paper is organized as follows. Section II, gives a general overview of the MR-FDPF method. In Section III, we describe the proposed modification of the MRFDPF method for channel modeling in OFDM systems. The MR-FDTLM method is described in Section IV. The results of the performance analysis for the proposed implementation of the MR-FDPF method are presented in Section V. The concluding remarks are given in Section VI.

\section{MR-FDPF METHOD}

In this section, a general overview of the MR-FDPF method is provided in order to facilitate the presentation of the material in Section III. For additional details, an interested reader is addressed to [5], [12], [13].

\section{A. ParFlow Formulation}

The ParFlow (or, more exactly, the time domain ParFlow) method is based on the cellular automata formalism [9], [14]-[16]. In this method, the scalar electric field strength is 


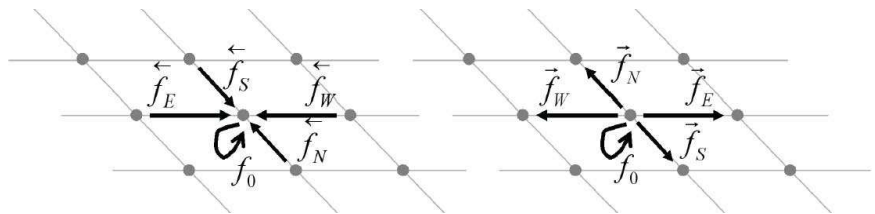

Fig. 1. Incident and reflected flows.

obtained by summing the fictitious flows traveling along a regular grid of connection lines and experiencing scattering at the nodes of the grid (see Fig. 1).

At every node $r$ of the grid, the discrete time evolution of the flows is described by the local scattering equation

$$
\overrightarrow{\mathbf{F}}(r, t)=\mathbf{W}(r) \overleftarrow{\mathbf{F}}(r, t-\Delta t)+\overrightarrow{\mathbf{S}}(r, t)
$$

where the incident (inward) flows vector $\overleftarrow{\mathbf{F}}(r, t)$, the reflected (outward) flows vector $\overrightarrow{\mathbf{F}}(r, t)$, and the source flows vector $\overrightarrow{\mathbf{S}}(r, t)$ are defined, respectively, as follows

$$
\begin{aligned}
& \overleftarrow{\mathbf{F}}(r, t)=\left[\overleftarrow{f}_{E}(r, t) \overleftarrow{f}_{W}(r, t) \overleftarrow{f}_{S}(r, t) \overleftarrow{f}_{N}(r, t) \breve{f}_{0}(r, t)\right]^{T} \\
& \overrightarrow{\mathbf{F}}(r, t)=\left[\vec{f}_{E}(r, t) \vec{f}_{W}(r, t) \vec{f}_{S}(r, t) \vec{f}_{N}(r, t) \breve{f}_{0}(r, t)\right]^{T} \\
& \overrightarrow{\mathbf{S}}(r, t)=\left[\vec{s}_{E}(r, t) \vec{s}_{W}(r, t) \vec{s}_{S}(r, t) \vec{s}_{N}(r, t) 0\right]^{T} .
\end{aligned}
$$

The operator $\{\cdot\}^{T}$ signifies matrix transposition. The inner flow $f_{0}(r, t)$ in $(2)$ models dielectric media with the relative permittivities $\varepsilon_{r} \neq 1$. The reflected flows at the grid node $r$ correspond to the incident flows at its immediate neighbor nodes, that is

$$
\overleftarrow{f}_{d}(r+\Delta r, t)=\vec{f}_{d}(r, t-\triangle t), \quad d \in E, W, S, N
$$

where $\triangle r$ is the spatial grid step, which is assumed to be the same along all the coordinate axes. The step $\triangle r$ satisfies the following conditions

$$
\triangle r=c_{0} \sqrt{2} \triangle t, \quad \triangle r \ll \lambda
$$

where $c_{0}$ denotes the speed of an electromagnetic wave in a propagation medium and $\lambda$ is the smallest wavelength in the spectrum of the source signal.

The scattering matrix $\mathbf{W}(r)$ in (1) reads as follows

$$
\mathbf{W}(r)=\left[\begin{array}{ccccc}
1 & \alpha_{r} & 1 & 1 & 1 \\
\alpha_{r} & 1 & 1 & 1 & 1 \\
1 & 1 & 1 & \alpha_{r} & 1 \\
1 & 1 & \alpha_{r} & 1 & 1 \\
Y_{r} & Y_{r} & Y_{r} & Y_{r} & \beta_{r}
\end{array}\right]
$$

where $\alpha_{r}=1-2 n_{r}^{2} ; \beta_{r}=2 n_{r}^{2}-4$. The parameters $n_{r}$ and $Y_{r}=4 n_{r}^{2}-4$ denote the refraction index and the local admittance, respectively. In order to satisfy the boundary conditions, the scattering matrix $\mathbf{W}(r)$ must be modified as described, e.g., in [9].

The complex scalar electric field strength $\Psi(r, t)$ is given by

$$
\Psi(r, t)=\frac{1}{2 n_{r}^{2}}\left(\breve{f}_{0}+\sum_{d=E, W, S, N} \vec{f}_{d}(r, t)\right) .
$$

\section{B. Multi-Resolution Frequency Domain (MR-FD) Approach}

Taking the Fourier transform of both sides of the local scattering equation (1) leads to

$$
\overrightarrow{\mathbf{F}}(r, \nu)=\mathbf{W}(r) e^{-j 2 \pi \nu \Delta t} \overleftarrow{\mathbf{F}}(r, \nu)+\overrightarrow{\mathbf{S}}(r, \nu)
$$

where $\nu$ denotes the frequency.

By aggregating the incident flow vectors $\overleftarrow{\mathbf{F}}(r, \nu)$ for all nodes into a single incident flow vector $\underline{\overleftarrow{\mathbf{F}}}(\nu)$ and similarly gathering the reflected flow vectors $\overrightarrow{\mathbf{F}}(r, \nu)$ and the source flow vectors $\overrightarrow{\mathbf{S}}(r, \nu)$ into the global flow vectors $\underline{\overrightarrow{\mathbf{F}}}(\nu)$ and $\underline{\mathbf{S}}(\nu)$, respectively, we come to the global scattering equation

$$
\underline{\mathbf{F}}(\nu)=\underline{\mathbf{W}} e^{-j 2 \pi \nu \Delta t} \underline{\overleftarrow{\mathbf{F}}}(\nu)+\underline{\mathbf{S}}(\nu)
$$

where $\underline{\mathbf{W}}$ is the global scattering matrix obtained by properly combining the local scattering matrices $\mathbf{W}(r)$ defined in (5).

Taking into account the relationship between the incident and the reflected flows of the adjacent nodes expressed by (3), equation (8) can be rewritten in terms of the reflected flow vector ${ }^{1} \underline{\mathbf{F}}(\nu)$ as

$$
\underline{\overrightarrow{\mathbf{F}}}(\nu)=\underline{\widetilde{\mathbf{W}}} e^{-j 2 \pi \nu \Delta t} \underline{\overrightarrow{\mathbf{F}}}(\nu)+\underline{\overrightarrow{\mathbf{S}}}(\nu)
$$

where the matrix $\widetilde{\widetilde{W}}$ signifies a permutated global scattering matrix $\mathbf{W}$.

The solution to equation (9) can now be found as

$$
\overrightarrow{\mathbf{F}}(\nu)=\left(\mathbf{I}-\underline{\widetilde{\mathbf{W}}} e^{-j 2 \pi \nu \Delta t}\right)^{-1} \underline{\mathbf{S}}(\nu)
$$

where I denotes the identity matrix.

The multi-resolution approach [5] provides a computationally efficient way of solving the equation (9). The approach is based on combining all the grid nodes into a single large head node that encompasses the considered propagation environment. After imposing prespecified boundary conditions, the head node is decomposed back into the original grid nodes. The process of applying the multi-resolution approach is divided into three steps: preprocessing step, upward step, and downward step. Further details related to the implementation of the MR-FD approach can be found in [5], [12]. Here, it is sufficient to mention that the highest computational load is associated with the preprocessing step. For a propagation environment of dimensions $M \times M$ nodes the computational loads for the preprocessing step, the upward step, and the downward step are estimated as [12]:

- preprocessing step: $O\left\{52 \cdot M^{3}\right\}$;

- upward step ${ }^{2}: O\left\{3 \cdot M^{2}\right\}$;

- downward step: $O\left\{10 \cdot \log _{2}(M) \cdot M^{2}\right\}$.

\section{OPTIMIZATION OF MR-FDPF METHOD FOR MULTIFREQUENCY SIMULATIONS}

A major limitation of the MR-FD approach is that it provides a solution $\underline{\overrightarrow{\mathbf{F}}}(\nu)$ (or, equivalently, $\underline{\mathbb{\mathbf { F }}}(\nu))$ of the global scattering equation (9) only at one frequency $\nu$. That means that the MR-FDPF method has to be applied repeatedly at

\footnotetext{
${ }^{1}$ Similarly, equation (8) can be reformulated in terms of the incident flow vector $\overleftarrow{\mathbf{F}}(\nu)$

${ }^{2}$ The computational cost is given per signal source.
} 
every frequency point in the range of interest $B$, e.g., for every sub-channel carrier frequency in OFDM communication systems.

In this section, a computationally efficient implementation of the MR-FDPF method is presented. The proposed implementation allows for a significant reduction in the computational load as compared to a straightforward repetition of the MR-FDPF method at multiple frequencies at the cost of acceptable degradation of the accuracy in predicting the electromagnetic wave propagation.

Without any loss of generality, we consider that only one signal source is present. We can also assume that $\underline{\overrightarrow{\mathbf{S}}}(\nu)=\underline{\mathbf{S}}$ $(\overrightarrow{\mathbf{S}} \neq 0)$, for all $\nu \in B$.

A solution (10) of equation (9) at the frequency $\nu_{0} \in B$ is given by

$$
\underline{\mathbf{F}}\left(\nu_{0}\right)=\left(\mathbf{I}-\underline{\widetilde{\mathbf{W}}}\left(\nu_{0}\right)\right)^{-1} \underline{\overrightarrow{\mathbf{s}}}
$$

where $\widetilde{\mathbb{\mathbf { W }}}\left(\nu_{0}\right)=\widetilde{\widetilde{\mathbf{W}}} e^{-j 2 \pi \nu_{0} \Delta t}$. Similar expression for the frequency $\nu_{0}+\Delta \nu$ reads as

$$
\overrightarrow{\mathbf{F}}\left(\nu_{0}+\Delta \nu\right)=\left(\mathbf{I}-\underline{\widetilde{\mathbf{W}}}\left(\nu_{0}+\Delta \nu\right)\right)^{-1} \underline{\mathbf{S}} .
$$

Note that $\underline{\widetilde{\mathbf{W}}}\left(\nu_{0}+\Delta \nu\right)=e^{-j 2 \pi \Delta \nu \Delta t} \underline{\widetilde{\mathbf{W}}}\left(\nu_{0}\right)$. Equation (12) can be rewritten as

$$
\begin{aligned}
& \overrightarrow{\mathbf{F}}\left(\nu_{0}+\Delta \nu\right)= \\
& \quad\left(\mathbf{I}-\underline{\widetilde{\mathbf{W}}}\left(\nu_{0}\right)+\underline{\widetilde{\mathbf{W}}}\left(\nu_{0}\right)-e^{-j 2 \pi \Delta \nu \Delta t} \underline{\widetilde{\mathbf{W}}}\left(\nu_{0}\right)\right)^{-1} \underline{\overrightarrow{\mathbf{S}}}
\end{aligned}
$$

After inserting (11) into (13) we obtain

$$
\overrightarrow{\mathbf{F}}\left(\nu_{0}+\Delta \nu\right)=\left(\mathbf{I}-\left(\mathbf{I}-\underline{\widetilde{\mathbf{W}}}\left(\nu_{0}\right)\right)^{-1} \underline{\widetilde{\mathbf{W}}}_{\Delta \nu}\right)^{-1} \underline{\overrightarrow{\mathbf{F}}}\left(\nu_{0}\right)
$$

where the matrix $\underline{\widetilde{\mathbf{W}}}_{\triangle \nu}=\left(e^{-j 2 \pi \Delta \nu \Delta t}-1\right) \underline{\widetilde{\mathbf{W}}}\left(\nu_{0}\right)$.

Using the Neumann series expansion (see, e.g., [17]) we can write

$$
\overrightarrow{\mathbf{F}}\left(\nu_{0}+\Delta \nu\right)=\left(\sum_{n=0}^{\infty}\left[\left(\mathbf{I}-\underline{\widetilde{\mathbf{W}}}\left(\nu_{0}\right)\right)^{-1} \underline{\widetilde{\mathbf{W}}}_{\triangle \nu}\right]^{n}\right) \overrightarrow{\mathbf{F}}\left(\nu_{0}\right)
$$

where it is implied that $\mathbf{A}^{0}=\mathbf{I}$ for any square matrix $\mathbf{A}$.

According to (15), an $N$-th order approximation of (12) is given by

$$
\underline{\overrightarrow{\mathbf{F}}}\left(\nu_{0}+\Delta \nu\right) \approx \underline{\overrightarrow{\mathbf{F}}}\left(\nu_{0}\right)+\underline{\overrightarrow{\mathbf{F}}}^{(1)}(\Delta \nu)+\ldots+\underline{\overrightarrow{\mathbf{F}}}^{(N)}(\Delta \nu)
$$

where

$$
\underline{\overrightarrow{\mathbf{F}}}^{(1)}(\triangle \nu)=\left(\mathbf{I}-\underline{\widetilde{\mathbf{W}}}\left(\nu_{0}\right)\right)^{-1} \underline{\widetilde{\mathbf{W}}}_{\triangle \nu} \underline{\overrightarrow{\mathbf{F}}}\left(\nu_{0}\right)
$$

and

$$
\underline{\overrightarrow{\mathbf{F}}}^{(n+1)}(\triangle \nu)=\left(\mathbf{I}-\underline{\widetilde{\mathbf{W}}}\left(\nu_{0}\right)\right)^{-1} \underline{\widetilde{\mathbf{W}}}_{\triangle \nu} \underline{\overrightarrow{\mathbf{F}}}^{(n)}(\triangle \nu)
$$

It is worth noticing that (18) (as well as (17)) is similar to (11). The only difference is that the source vector $\underline{\vec{S}}$ in (11) is substituted by the vector $\widetilde{\mathbf{W}}_{\triangle \nu} \overrightarrow{\mathbf{F}}^{(n)}(\triangle \nu)$ in (18). Thus, the MR-FDPF method can readily be applied in order to find the vector $\underline{\mathbf{F}}^{(n+1)}(\Delta \nu)$ in (18).

An important question is the convergence speed of the approximation (16). A sufficient condition for the convergence

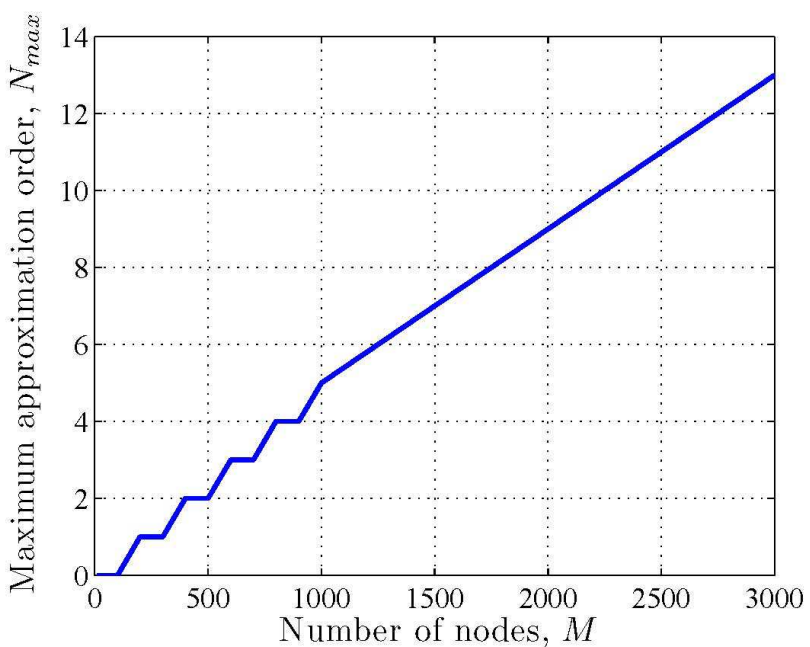

Fig. 2. The upper bound on the approximation order $N$ in (16), plotted as a function of the environment size $M$.

of the Neumann series is that the spectral radius of the matrix $\left(\mathbf{I}-\underline{\widetilde{\mathbf{W}}}\left(\nu_{0}\right)\right)^{-1}\left(e^{-j 2 \pi \Delta \nu \Delta t}-1\right) \underline{\widetilde{\mathbf{W}}}\left(\nu_{0}\right)$ is less than 1 [17]. Based on the fact that in practice, the eigenvalues of the scattering matrix $\underline{\widetilde{\mathbf{W}}}(\nu)$ must be real and smaller than 1 , it can be induced that the smaller the spectral radius of the matrix $\underline{\mathbf{W}}(\nu)$ (or, in different words, the larger the attenuation due to the propagation environment), the faster is the convergence of the approximation (16).

The advantage of using the approximation (16) lies in the fact that the scattering matrix $\underline{\mathbf{W}}(\nu)$ is calculated only for the frequency $\nu_{0}$. That means that the preprocessing step (see [5], [12]), which is the most computationally expensive one, is conducted only once. On the downside, however, equation (18) implies that while calculating the terms $\overrightarrow{\mathbf{F}}^{(n)}(\Delta \nu), n=$ $1,2, \ldots$, each node is a signal source. Thus, the computational cost of the upward step for each additional term $\overrightarrow{\mathbf{F}}^{(n)}(\Delta \nu)$ becomes $O\left\{\log _{2}(M) M^{2}\right\}$ (up to a constant scaling factor). Of course, the additional computational loads associated with the upward step and the downward step are linearly proportional to the number of terms $N$ in the approximation (16). In this connection, the number of terms $N$ is limited by the inequality

$$
N<N_{\max }=1+\left\lfloor\frac{\mathcal{C}_{p}(M)}{\mathcal{C}_{u d}(M)}\right\rfloor
$$

where $\mathcal{C}_{p}(M)$ represents the computational complexity of the preprocessing step and $\mathcal{C}_{u d}(M)$ is the cumulative cost of the upward step and the downward step. The operator $\lfloor x\rfloor$ denotes the largest integer less than or equal to $x$.

The graph in Fig. 2 illustrates the upper bound $N_{\max }$ defined in (19). The curve in the figure is plotted based on the computational costs for the preprocessing step, the upward step, and the downward step provided in Subsection II-B. It follows from the graph that for propagation environments of size $M \geq 2000$ nodes, the computational cost of the preprocessing step is dominating, hence we can expect computational savings from using the approximate solution (16). Note, however, that the results in Fig. 2 can be considered only as rough estimates 
of the upper bound $N_{\max }$. (Observe the big O notation in the evaluations of the computational costs of the preprocessing, upward, and downward steps.)

In Section V, we assess the performance of the proposed implementation of the MR-FDPF method in terms of computational load and accuracy based on the simulation results obtained for two propagation scenarios. But before that, in Section IV, we consider application of the MR-FD approach to the well-known TLM method.

\section{MR-FD APPROACH FOR TLM METHOD}

Perhaps the most attractive feature of the ParFlow approach is that the local scattering matrices $\mathbf{W}(r)$ defined in (1) are completely determined by the parameters of corresponding propagation media and are independent of the simulation time step and the spatial mesh size. Unfortunately, the ParFlow approach cannot be readily extended to simulate electromagnetic wave propagation in $3 \mathrm{D}$ space.

On the other hand, similar to the ParFlow formulation, the representation of a propagation medium by means of the scattering matrix defined for each node of the mesh can be found in the TLM method (see, e.g., [10]). In contrast to the ParFlow approach, the 3D TLM method is readily available. Thus, it is of interest to investigate if the MR-FD method implementation presented in the previous section can be applied to the TLM method, particularly in 3D space.

\section{A. TLM Method Formulation}

In the TLM method, the wave propagation environment is modeled as an aggregation of transmission lines interconnected at nodes. In 3D space (the most general case), considered below, the nodes are constructed by interconnecting 12 transmission lines and up to 6 stubs [18]. The stubs are introduced into the nodes to allow modeling of inhomogeneous propagation media on a graded mesh. In the following, we consider the nodes constructed only by interconnecting the transmission lines, which are called symmetrical supper-condensed nodes (SSCNs) [18]. Our choice of the SSCN is mainly dictated by the absence of stubs, which means that the size of the local scattering matrix is $12 \times 12$, while the stub-loaded symmetrical condensed nodes (SCNs) generate local scattering matrices of dimensions up to ${ }^{3} 18 \times 18(15 \times 15$ for hybrid SCNs) [18]. A detailed description of the TLM method and the SSCNs can be found in [2], [18]-[21] and the multiple references therein.

In the TLM method, the incident and reflected voltage pulses travel along the transmission lines, which are characterized by the line impedances $Z$ and conductivities $Y$. Similar to the ParFlow formulation (see Section II), at every node $r$, the local scattering equation is defined as ( $c f .(1))$

$$
\overrightarrow{\mathbf{V}}(r, t)=\mathbf{U}(r) \overleftarrow{\mathbf{V}}(r, t-\Delta t)+\overrightarrow{\mathbf{S}}(r, t)
$$

where the vectors $\overleftarrow{\mathbf{V}}(r, t), \overrightarrow{\mathbf{V}}(r, t)$, and $\overrightarrow{\mathbf{S}}(r, t)$, of dimensions $12 \times 1$, denote, respectively, the incident, reflected, and

\footnotetext{
${ }^{3}$ As there are no connections between the stubs of neighboring nodes, the signals related to the stubs can be treated similar to the inner flows in (2). That means that in the MR-FDTLM method the size of the local scattering matrices for all types of SCNs can be reduced to $12 \times 12$ at no additional computational cost (for details, see [5]).
}

excitation voltage pulses. The matrix $\mathbf{U}(r)$ is the SSCN local scattering matrix of dimensions $12 \times 12$ (see [18]).

Due to the fact that the line impedance experiences a discontinuity at the interfaces between two nodes belonging to two different media, the partial reflections from the junction have to be taken into account while calculating the results of the scattering at the nodes [18].

\section{B. Application of MR-FD Approach to TLM Method (MR- FDTLM)}

It appears that the only impediment that prevents a direct application of the MR-FD approach to the TLM method is the reflections/transmissions occurring at the interfaces between different node regions, i.e., at the boundaries between different propagation media. As it is shown below, this impediment can be removed.

For this purpose, it is more convenient to represent the scattering equation (20) in the state-space form

$$
\begin{aligned}
\mathbf{x}(r, t+\Delta t) & =\mathbf{R}(r) \mathbf{x}(r, t)+\mathbf{U}(r) \overleftarrow{\mathbf{V}}(r, t) \\
\overrightarrow{\mathbf{V}}(r, t) & =\mathbf{T}(r) \mathbf{x}(r, t)+\overrightarrow{\mathbf{S}}(r, t) .
\end{aligned}
$$

where the vectors $\overleftarrow{\mathbf{V}}(r, t), \overrightarrow{\mathbf{V}}(r, t), \overrightarrow{\mathbf{S}}(r, t)$ and the scattering matrix $\mathbf{U}(r)$ have been defined above (see (20)). The vector $\mathbf{x}(r, t)$ in (21) represents the state variables. The matrices $\mathbf{R}(r)$ and $\mathbf{T}(r)$ are diagonal.

The diagonal elements $R_{i i}(r)$ of the reflection matrix $\mathbf{R}(r)$ are given by

$$
R_{i i}(r)=\frac{Z_{j}(r+\triangle r)-Z_{i}(r)}{Z_{j}(r+\triangle r)+Z_{i}(r)}
$$

where $Z_{j}(r+\Delta r)$ and $Z_{i}(r), i, j=1 \ldots 12$, are the impedances of the transmission lines connecting the neighbor nodes ${ }^{4}$ located at $r$ and $r+\Delta r$. Note that when both nodes at $r$ and $r+\Delta r$ belong to the same medium, the corresponding diagonal elements of the matrices $\mathbf{R}(r)$ and $\mathbf{R}(r+\triangle r)$ equal zero. Thus, if the node at $r$ is surrounded by the nodes of the same medium, the reflection matrix $\mathbf{R}(r)$ is zero.

The diagonal elements $T_{i i}^{p}$, of the transmission matrix $\mathbf{T}(r)$ are defined as

$$
T_{i i}(r)=\frac{2 Z_{i}(r)}{Z_{j}(r+\triangle r)+Z_{i}(r)} .
$$

When both nodes positioned at $r$ and $r+\Delta r$ belong to the same medium, the corresponding diagonal elements of the matrices $\mathbf{T}(r)$ and $\mathbf{T}(r+\Delta r)$ are 1 . Thus, the transmission matrix $\mathbf{T}(r)$ becomes the identity matrix $\mathbf{I}$, when the node at $r$ is surrounded by the nodes of the same medium.

By taking the Fourier transform of (21) and after straightforward manipulations we obtain

$$
\begin{aligned}
& \overrightarrow{\mathbf{V}}(r, \nu)= \\
& \mathbf{T}(r)\left(\mathbf{I}-\mathbf{R}(r) e^{-j 2 \pi \nu \Delta t}\right)^{-1} e^{-j 2 \pi \nu \Delta t} \mathbf{U}(r) \overleftarrow{\mathbf{V}}(r, \nu)+ \\
& \quad+\overrightarrow{\mathbf{S}}(r, \nu)=\boldsymbol{\Sigma}(r, \nu) \overleftarrow{\mathbf{V}}(r, \nu)+\overrightarrow{\mathbf{S}}(r, \nu)
\end{aligned}
$$

${ }^{4}$ For a uniformly spaced grid, which is assumed here, the spatial grid step $\triangle r$ is the same along all the coordinate axes, i.e., $\triangle x=\Delta y=\Delta z=\Delta r$ (see also Section II). 
which is analogous to the frequency domain ParFlow formulation in (7).

On gathering the local vectors $\overleftarrow{\mathbf{V}}(r, \nu), \overrightarrow{\mathbf{V}}(r, \nu)$, and $\overrightarrow{\mathbf{S}}(r, \nu)$ for all nodes into the global vectors $\underline{\mathbf{V}}(\nu), \underline{\mathbf{V}}(\nu)$, and $\underline{\overrightarrow{\mathbf{S}}}(\nu)$, respectively, we can write the global scattering equation

$$
\underline{\overrightarrow{\mathbf{V}}}(\nu)=\underline{\boldsymbol{\Sigma}}(\nu) \underline{\overleftarrow{\mathbf{V}}}(\nu)+\underline{\overrightarrow{\mathbf{S}}}(\nu)
$$

where the matrix $\boldsymbol{\Sigma}(\nu)$ is the global scattering matrix obtained by combining the local scattering matrices $\boldsymbol{\Sigma}(r, \nu)$.

Note that as the equation (25) is per se the Fourier transform of (20), the stability and the dispersion characteristics of the MR-FDTLM method remain the same as compared to the similar characteristics of the underlying TLM method.

\section{Computational Complexity of MR-FDTLM Method}

As it has been pointed out above, the MR-FD approach (see Section II) provides a computationally efficient way of solving the equation (25) with respect to the vectors $\underline{\mathbf{V}}(\nu)$ (or, equivalently, $\underline{\mathbb{\mathrm { V }}}(\nu)$ ).

In order to assess the computational complexity of the MRFDTLM method, we presume that it is mainly determined by the computational cost of the matrix manipulations carried out in the course of a recursive composition of scattering blocks (see [5]). For completeness of the presentation, the composition of two scattering blocks is described in the Appendix. The described procedure is a slightly modified version of the algorithm considered in [22]. To simplify the assessment of the computational complexity of the MR-FDTLM method, we assume that the propagation environment is a cube of dimensions $M \times M \times M$ SSCN nodes, where $M=2^{L}$.

In agreement with the notation used in Section II (see also [12]), the matrix manipulations in (43) can be identified as the preprocessing step, while (44) and (41) correspond to the upward step and the downward step, respectively. The total computational costs of the preprocessing step, upward step, and downward step are estimated as

- preprocessing step: $O\left\{41472 \cdot M^{6}\right\}$;

- upward step: $O\left\{2304 \cdot M^{4}\right\}$;

- downward step: $O\left\{4608 \cdot M^{4}\right\}$.

Observe that the computational load for the preprocessing step dominates those associated with the upward step and the downward step in the whole range of possible values of $M$. Thus, similar to the approach presented in Section III for the MR-FDPF method, it is desirable to reduce the number of performed preprocessing steps in order to lower the computational complexity of the MR-FDTLM method employed for simulations at multiple frequencies.

\section{MR-FDTLM Method for Multifrequency Simulations}

Clearly, the form of the the global scattering equation (25) for the MR-FDTLM method is similar to the equation (9) given in Section III for MR-FDPF method. Note, however, that in contrast to the matrix $\underline{\widetilde{\mathbf{W}}}\left(\nu_{0}+\Delta \nu\right)$ in (12), the global scattering matrix $\boldsymbol{\Sigma}(\nu+\Delta \nu)$, in general, cannot be written as $e^{-j 2 \pi \Delta \nu \Delta t} \underline{\boldsymbol{\Sigma}}\left(\nu_{0}\right)$ (see expression (24)). Thus, the procedure described in Section III has to be modified in order to be applicable to the MR-FDTLM method.

Let us again consider the local scattering matrix $\boldsymbol{\Sigma}(r, \nu)$ in (24). Suppose that the matrix $\boldsymbol{\Sigma}\left(r, \nu_{0}\right)$ corresponding to the frequency $\nu_{0} \in B$ is known for each node. Then, assuming that $\triangle \nu$ is sufficiently small, we can use a first-order Taylor expansion to approximate the matrix $\boldsymbol{\Sigma}(r, \nu)$ at the frequency $\nu=\nu_{0}+\triangle \nu \in B$ as follows

$$
\begin{aligned}
& \boldsymbol{\Sigma}(r, \nu) \approx \\
& \quad \boldsymbol{\Sigma}\left(r, \nu_{0}\right)+\left.\frac{d \boldsymbol{\Sigma}(r, \nu)}{d \nu}\right|_{\nu=\nu_{0}} \Delta \nu=\boldsymbol{\Sigma}\left(r, \nu_{0}\right)+\boldsymbol{\Sigma}_{\triangle \nu}(r) .
\end{aligned}
$$

It can be shown that

$$
\begin{aligned}
& \frac{d \boldsymbol{\Sigma}(r, \nu)}{d \nu}= \\
& \quad j 2 \pi \Delta t e^{j 2 \pi \nu \Delta t} \mathbf{T}(r)\left(e^{j 2 \pi \nu \Delta t} \mathbf{I}-\mathbf{R}(r)\right)^{-2} \mathbf{U}(r)
\end{aligned}
$$

and

$$
\begin{aligned}
& \boldsymbol{\Sigma}_{\triangle \nu}(r)= \\
& \quad j 2 \pi \triangle t \triangle \nu e^{j 2 \pi \nu_{0} \Delta t} \mathbf{T}(r)\left(e^{j 2 \pi \nu_{0} \triangle t} \mathbf{I}-\mathbf{R}(r)\right)^{-2} \mathbf{U}(r) .
\end{aligned}
$$

We can now reformulate the global scattering equation (25) as follows

$$
\begin{aligned}
& \underline{\overrightarrow{\mathbf{V}}}(\nu+\Delta \nu)=\underline{\boldsymbol{\Sigma}}(\nu+\Delta \nu) \underline{\overleftarrow{\mathbf{V}}}(\nu+\Delta \nu)+\underline{\overrightarrow{\mathbf{S}}}(\nu+\Delta \nu) \\
& \quad \approx\left(\underline{\boldsymbol{\Sigma}}\left(\nu_{0}\right)+\underline{\boldsymbol{\Sigma}}_{\triangle \nu}\right) \underline{\overleftarrow{\mathbf{V}}}(\nu+\Delta \nu)+\underline{\overrightarrow{\mathbf{S}}}(\nu+\Delta \nu)
\end{aligned}
$$

where $\underline{\boldsymbol{\Sigma}}\left(\nu_{0}\right)+\underline{\boldsymbol{\Sigma}}_{\triangle \nu}$ is an approximation of the global scattering matrix $\underline{\boldsymbol{\Sigma}}(\nu)$.

Presuming that $\underline{\overrightarrow{\mathbf{s}}}(\nu)=\underline{\overrightarrow{\mathbf{s}}} \neq 0$, for all $\nu \in B$, and taking into account the relationship between the incident and reflected voltage pulses of the adjacent nodes, which is similar to equation (3), we obtain

$$
\begin{aligned}
\underline{\overrightarrow{\mathbf{V}}}(\nu+\Delta \nu) & =(\mathbf{I}-\underline{\tilde{\boldsymbol{\Sigma}}}(\nu))^{-1} \underline{\overrightarrow{\mathbf{S}}} \\
& \approx\left(\mathbf{I}-\underline{\tilde{\boldsymbol{\Sigma}}}\left(\nu_{0}\right)-\underline{\widetilde{\boldsymbol{\Sigma}}}_{\triangle \nu}\right)^{-1} \underline{\overrightarrow{\mathbf{S}}}
\end{aligned}
$$

where $\underline{\tilde{\boldsymbol{\Sigma}}}\left(\nu_{0}\right)$ and $\underline{\widetilde{\boldsymbol{\Sigma}}}_{\triangle \nu}$ are the permutated matrices $\underline{\boldsymbol{\Sigma}}\left(\nu_{0}\right)$ and $\underline{\boldsymbol{\Sigma}}_{\triangle \nu}$, respectively. The second part of equation (30) can be redefined as

$$
\overrightarrow{\underline{\mathbf{V}}}(\nu+\Delta \nu) \approx\left(\mathbf{I}-\left(\mathbf{I}-\underline{\tilde{\Sigma}}\left(\nu_{0}\right)\right)^{-1} \underline{\widetilde{\Sigma}}_{\Delta \nu}\right)^{-1} \underline{\overrightarrow{\mathbf{V}}}\left(\nu_{0}\right) .
$$

where

$$
\underline{\overrightarrow{\mathbf{V}}}\left(\nu_{0}\right)=\left(\mathbf{I}-\underline{\tilde{\mathbf{s}}}\left(\nu_{0}\right)\right)^{-1} \underline{\mathbf{S}}
$$

is the exact solution of the global scattering equation (30) at the frequency $\nu_{0}$.

Similar to equation (15)-(18), we can write

$$
\underline{\overrightarrow{\mathbf{V}}}\left(\nu_{0}+\Delta \nu\right) \approx\left(\sum_{n=0}^{\infty}\left[\left(\mathbf{I}-\underline{\tilde{\boldsymbol{\Sigma}}}\left(\nu_{0}\right)\right)^{-1} \underline{\widetilde{\boldsymbol{\Sigma}}}_{\triangle \nu}\right]^{n}\right) \underline{\overrightarrow{\mathbf{V}}}\left(\nu_{0}\right)
$$

and, consequently,

$$
\underline{\overrightarrow{\mathbf{V}}}\left(\nu_{0}+\Delta \nu\right) \approx \underline{\overrightarrow{\mathbf{V}}}\left(\nu_{0}\right)+\underline{\overrightarrow{\mathbf{V}}}^{(1)}(\Delta \nu)+\underline{\overrightarrow{\mathbf{V}}}^{(2)}(\triangle \nu)+\ldots
$$




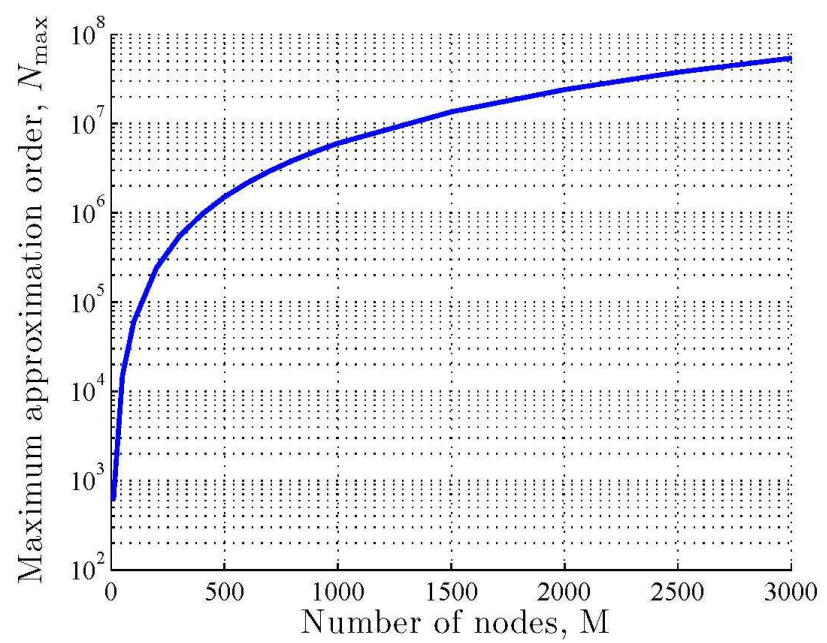

Fig. 3. The upper bound on the approximation order $N$ in (34), plotted as a function of the environment size $M$.

where

$$
\begin{array}{r}
\underline{\overrightarrow{\mathbf{V}}}^{(1)}(\Delta \nu)=\left(\mathbf{I}-\underline{\tilde{\boldsymbol{\Sigma}}}\left(\nu_{0}\right)\right)^{-1} \underline{\widetilde{\boldsymbol{\Sigma}}}_{\Delta \nu} \underline{\overrightarrow{\mathbf{V}}}\left(\nu_{0}\right) \\
\underline{\overrightarrow{\mathbf{V}}}^{(n+1)}(\Delta \nu)=\left(\mathbf{I}-\underline{\tilde{\boldsymbol{\Sigma}}}\left(\nu_{0}\right)\right)^{-1} \underline{\widetilde{\boldsymbol{\Sigma}}}_{\triangle \nu} \underline{\overrightarrow{\mathbf{V}}}^{(n)}(\Delta \nu) .
\end{array}
$$

The same line of reasoning regarding the convergence speed as in Section III can now be applied to the approximation (34).

Evidently, the goal to reduce the computational complexity of the MR-FDTLM method in multifrequency simulations is achieved when the number of terms in the approximation (34) does not exceed the bound $N_{\max }$ previously defined in (19). The parameter $N_{\max }$ as a function of the size $M$ of a propagation environment is illustrated by the graph in Fig. 3.

Although indirectly, relatively large values of $N_{\max }$ ( $N_{\max }>10^{2}$ for $M \geq 15$ ) in Fig. 3 point to a considerable potential of the method implementation proposed in this subsection in cutting down the computational load of the MR-FDTLM in multifrequency simulations. Again, we have to remark that the graph in Fig. 3 represents only rough estimates of the upper bound $N_{\max }$.

\section{EXPERIMENTAL RESUlTS}

In this section, we present the results of applying the proposed implementation of the MR-FDPF approach to predicting radio coverage in two realistic propagation scenarios. Unfortunately, we are not able to provide any experimental results for the MR-FDTLM method described in Section IV as, presently, no TLM based prediction tool is at our disposal.

\section{A. Propagation Scenarios}

The first propagation scenario is inside the CITI building, INSA university campus in Lyon. The building dimensions are approximately $40 \times 60 \mathrm{~m}$. The electric field strength $\Psi(r, \nu)$ inside the CITI building and its immediate surroundings has been computed with a spatial resolution of $2 \mathrm{~cm}$. In Fig. 4, the power $P(r, \nu)$ of the electric field predicted by the MRFDPF method at the frequency $\nu=2.345 \mathrm{GHz}$ is depicted.

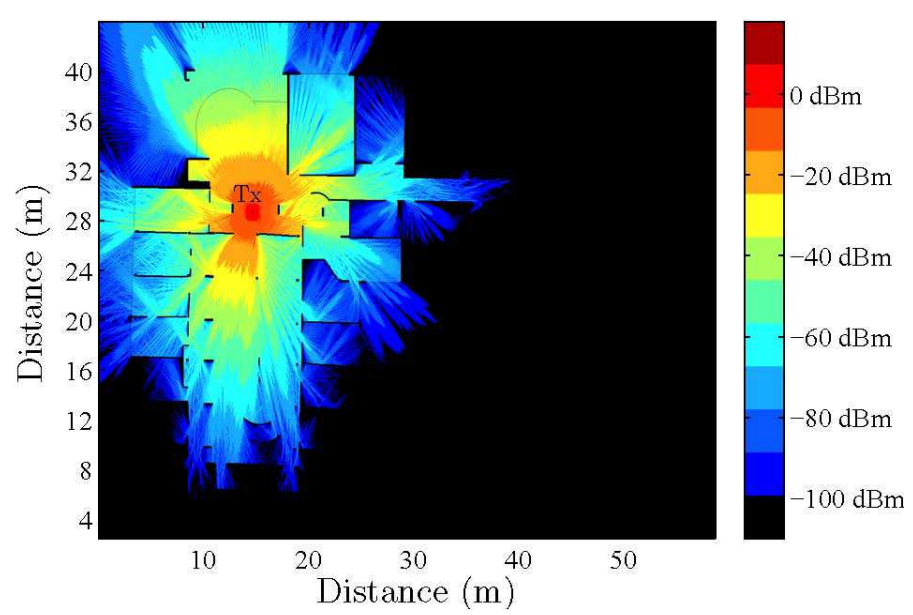

Fig. 4. CITI. The electric field power $P(r, \nu)$ predicted at the frequency $\nu=2.345 \mathrm{GHz}$.

The position of the omnidirectional transmitting antenna is indicated in Fig. 4.

The second propagation scenario is an indoor office environment in Stanford University. The dimensions of the environment are approximately $16 \times 34 \mathrm{~m}$. The power $P(r, \nu)$ of the electric field predicted by the MR-FDPF method at the frequency $\nu=2.345 \mathrm{GHz}$ is shown in Fig. 5. The position of the transmitter equipped with omnidirectional antenna is also depicted in Fig. 5. The electric field strength $\Psi(r, \nu)$ has been calculated with the $2 \mathrm{~cm}$ spatial resolution.

In the following analysis, the locations $r$ where the predicted power $P(r, \nu)$ of the electric field is below $-100 \mathrm{dBm}$ (black areas in Figs. 4 and 5) are not taken into account, i.e., the dynamic range is limited to approximately $100 \mathrm{~dB}$.

\section{B. Convergence of the approximation}

In this subsection, we verify the convergence of the approximation (16) as the number of terms $N$ increases.

Figs. 6 and 7 show the mean absolute power approximation errors $|\hat{P}(r, \nu)[d B m]-P(r, \nu)|[d B m]$ obtained for the two considered propagation environments, respectively. Here, $\hat{P}(r, \nu)$ denotes the electric field power predicted with the MR-FDPF method optimized for multifrequency simulations, as described in Section III, and $P(r, \nu)$ is the electric field power predicted with the traditional implementation of the MR-FDPF method, i.e., the implementation where the preprocessing step takes place at every frequency $\nu$. As expected, a larger deviation $\triangle \nu$ from the central frequency $\nu_{0}$ causes the absolute approximation error to expand. Also, as the number of terms $N$ in (16) increases, the average absolute approximation error decreases, or, alternatively, we observe expansion of the frequency band, in which the error is bounded by a predefined level, e.g., $1 \mathrm{~dB}$. Somewhat unexpectedly, the mean absolute power approximation errors tend to be symmetrical with respect to (w.r.t.) the central frequency $\nu_{0}$ for both propagation scenarios. However, it appears that this tendency is a result of averaging as it does not apply to every location $r$ (see Figs. 8 and 9 below). 


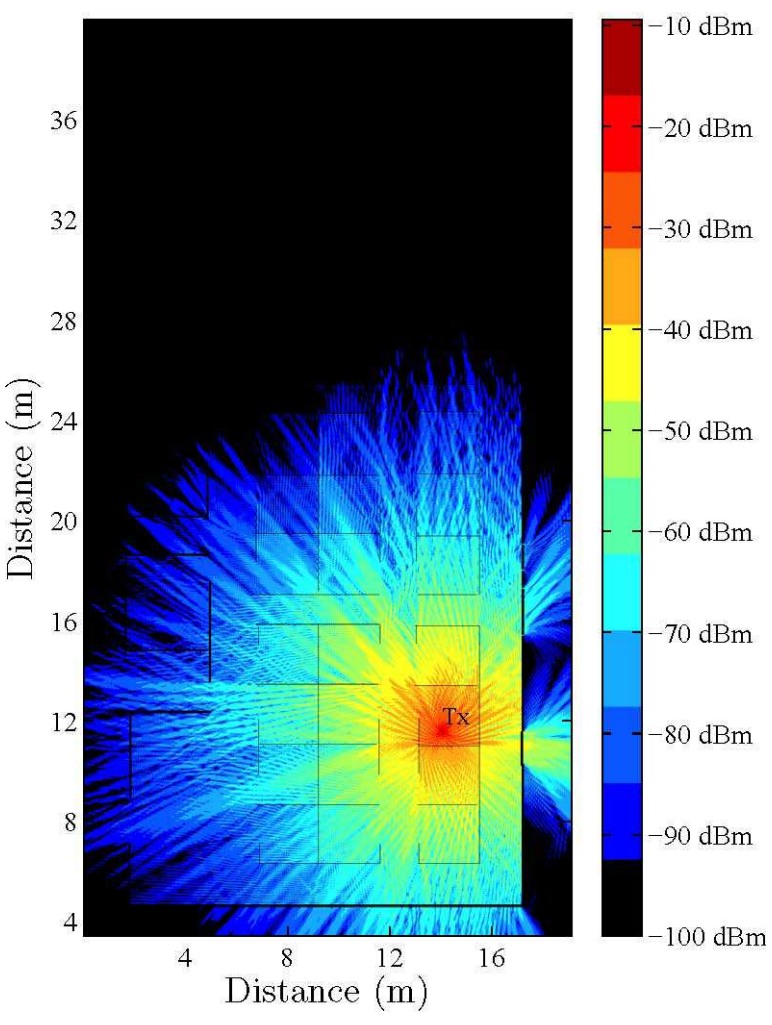

Fig. 5. Stanford. The electric field power $P(r, \nu)$ predicted at the frequency $\nu=2.345 \mathrm{GHz}$.

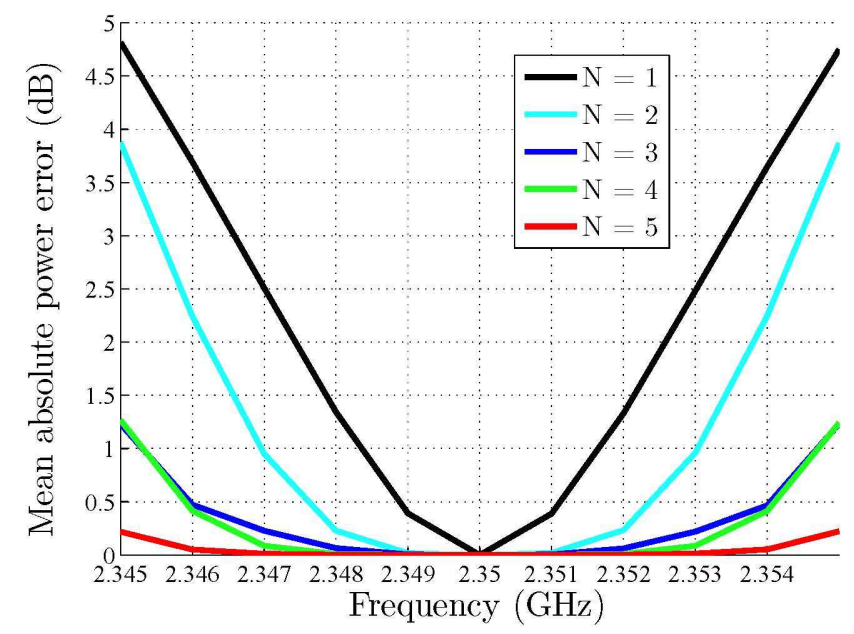

Fig. 6. CITI. Average absolute power approximation error $\left(\nu_{0}=2.35 \mathrm{GHz}\right)$.

Let us now turn to the computational efficiency of the proposed implementation of the MR-FDPF method.

\section{Computational Load}

The performance of the MR-FDPF implementation optimized for multifrequency simulations is measured against the performance of the traditional implementation of the method.

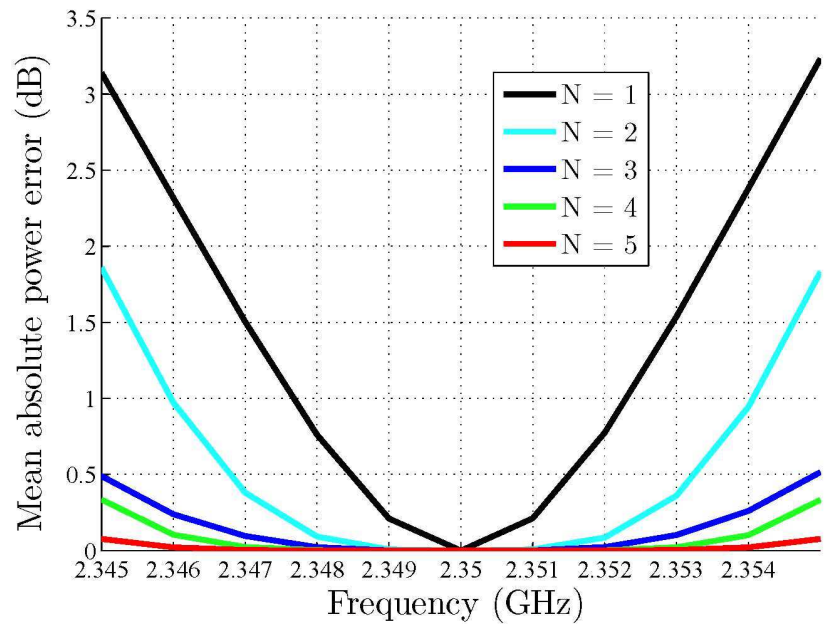

Fig. 7. Stanford. Average absolute power approximation error $\left(\nu_{0}=\right.$ $2.35 \mathrm{GHz}$ ).

We compare the time it takes to run the proposed and the traditional implementations on the same computer. The computer is a server running under a 64-bit Linux-type operating system. The maximum amount of RAM reserved for the application is 12 GB. It should be mentioned that the main body of the code has been written in Java. However, general linear matrix operations, e.g., matrix multiplication, have been implemented in $\mathrm{C}$. The code has been designed as a single thread application.

The experimental results pertaining to the two propagation scenarios are shown in Tables I and II, respectively. The performance of the traditional implementation of the MR-FDPF method has been taken as a reference, which corresponds to $100 \%$. It follows from Tables I and II that the approximation (16) with only one term $(N=1)$ provides roughly $60 \%$ and $50 \%$ reduction in the running time of the MR-FDPF method for the CITI environment and the Stanford environment, respectively. When $N=2$, the proposed implementation of the MR-FDPF method requires around $30 \%$ less time for the CITI environment and around $20 \%$ less time for the Stanford environment. Further increase of the number of terms in the approximation (16) is not practical for the considered propagation scenarios as the time needed to run the new implementation of the MR-FDPF method exceeds the running time required by the traditional implementation of the method.

TABLE I

CITI. Average RElative RUNNING TIME.

\begin{tabular}{|c|c|c|c|}
\hline & $N=1$ & $N=2$ & $N=3$ \\
\hline Computational time, (\%) & 43 & 70 & 103 \\
\hline
\end{tabular}

TABLE II

StANFord. AVERAGE RElative RUNNING TIME.

\begin{tabular}{|l|c|c|c|}
\hline & $N=1$ & $N=2$ & $N=3$ \\
\hline Computational time, (\%) & 51 & 82 & 109 \\
\hline
\end{tabular}

It is also of interest to compare the experimental results 
in Tables I and II with the upper bounds $N_{\max }$ predicted in Section III for propagation environments of similar sizes. According to Fig. 2, $N_{\max }=11$ for the CITI propagation environment $(M=2483)$ and $N_{\max }=6$ for the Stanford propagation environment $(M=1323)$, which substantially disagree (see Section III) with the values observed in practice. Worthy of note is that the values of $N_{\max }$ plotted in Fig. 2 do not reflect the overhead time consumed on shuffling the data between the different memory cashes. The amount of memory required for keeping the intermediate results is roughly doubled for the the proposed implementation of the MR-FDPF method as compared to the traditional implementation. Consequently, increased attention should be paid to the program code optimization in order to reduce the potential overhead.

\section{Frequency Domain Characteristics}

The 1-dB error relative bandwidths $(\% B)$ for the considered propagation environments are collected in Tables III and IV, respectively. The data are obtained based on the graphs in Figs. 6 and 7 presented above.

TABLE III

CITI. 1-DB ERROR RELATIVE BANDWIDTH.

\begin{tabular}{|c|c|c|}
\hline & $N=1$ & $N=2$ \\
\hline Relative bandwidth, (\%) & 0.1 & 0.25 \\
\hline
\end{tabular}

TABLE IV

STANFORD. 1-DB ERROR RELATIVE BANDWIDTH.

\begin{tabular}{|c|c|c|}
\hline & $N=1$ & $N=2$ \\
\hline Relative bandwidth, (\%) & 0.2 & 0.3 \\
\hline
\end{tabular}

In Figs. 8 and 9, we show two arbitrary chosen examples of the channel transfer functions corresponding to the considered propagation environments. The lines marked "MR-FDPF" in the figures correspond to the traditional implementation of the MR-FDPF method, while the lines "MR-FDPF MF" are related to the proposed implementation of the MR-FDPF method optimized for multifrequency simulations. The results presented in Figs. 8 and 9 are in agreement with Figs. 6 and 7 , i.e., the discrepancy between the channel transfer functions corresponding to the new and the traditional implementations of the MR-FDPF method decreases as the number of terms $N$ in the approximation (16) increases. It can be seen from Figs. 8 and 9 that the frequency response approximation errors (as well as the power approximation errors) are not symmetrical w.r.t. the central frequency $\nu_{0}$. Also note the approximation error can be of both positive and negative signs.

In order to get an overview of the performance of the proposed MR-FDPF method implementation over the frequency range at multiple locations, it is desirable to describe the predicted channel transfer functions by a single parameter. The two possible candidates for this role are the coherence bandwidth and the delay spread, which are widely used in practice to characterize frequency-selective channels. The coherence bandwidth would be particularly suitable for the

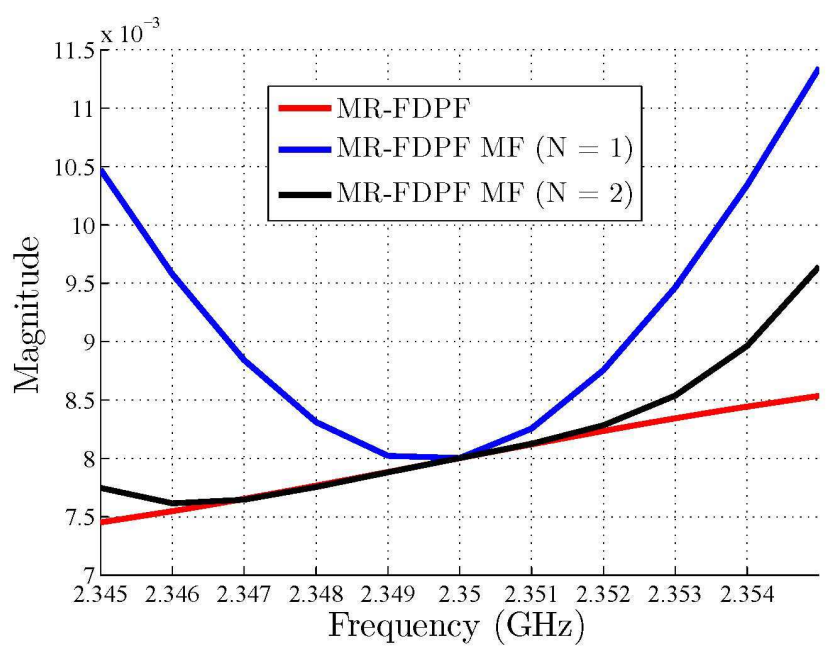

Fig. 8. CITI. The frequency response of the channel between the transmitter and the point with the coordinates $32.1 \mathrm{~m}$ and $21.54 \mathrm{~m}$ along the vertical and horizontal axes, respectively (see Fig. 4).

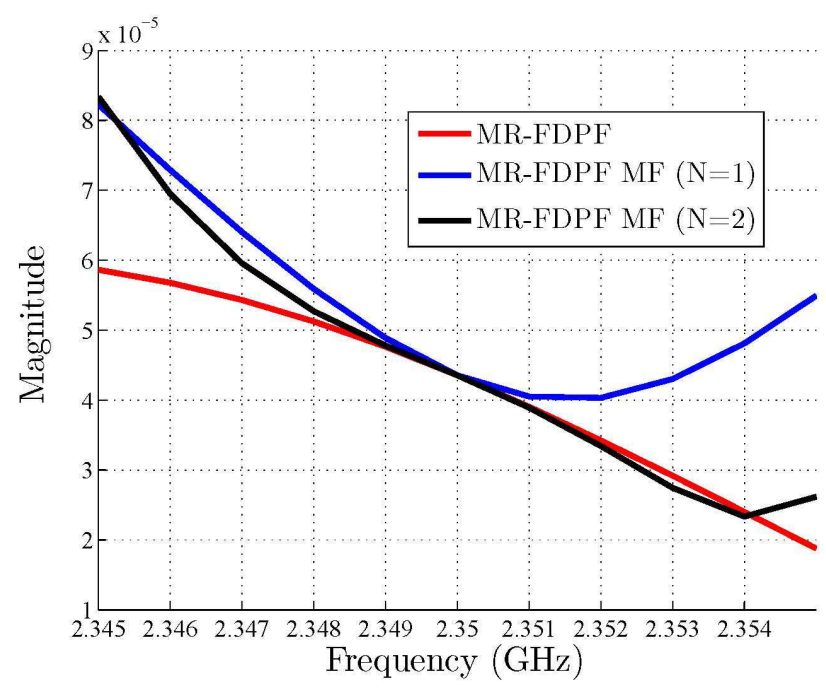

Fig. 9. Stanford. The frequency response of the channel between the transmitter and the point with the coordinates $11.1 \mathrm{~m}$ and $18.54 \mathrm{~m}$ along the vertical and horizontal axes, respectively (see Fig. 5).

OFDM systems see, e.g., [4]. However, as the frequency range is limited to $10 \mathrm{MHz}$ in our examples, it is rather difficult to select a single correlation threshold for all locations. Therefore, the delay spread defined, e.g., in [4], is appear to be the most appropriate. The description of the relationship between the delay spread and the coherence bandwidth can be found in [4], [23].

The delay spreads $S_{\tau}(r)$ and $\hat{S}_{\tau}(r)$ have been estimated based on the electric field strength predicted at the frequencies $\nu=2.345,2.346 \ldots, 2.355 \mathrm{GHz}$ with the traditional MRFDPF implementation and the implementation optimized for multifrequency simulations, respectively. In order to improve the estimates, the calculated power delay profiles (PDPs) have been averaged over $7 \times 7$ point regions centered at the selected positions. The delay spread relative absolute errors for the considered propagation scenarios, defined as 


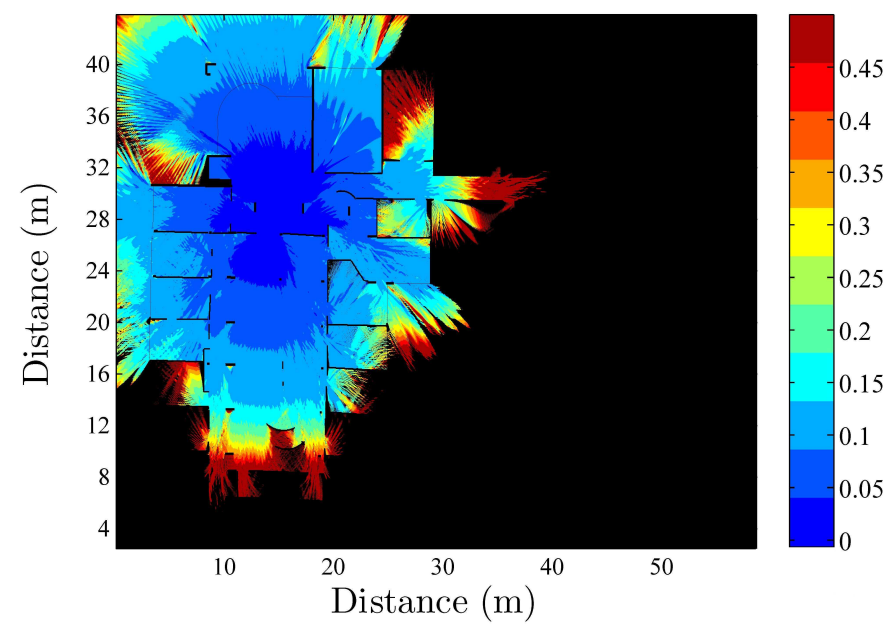

Fig. 10. CITI. The delay spread relative absolute error $\mid\left(S_{\tau}(r)-\right.$ $\left.\hat{S}_{\tau}(r)\right) / S_{\tau}(r) \mid(N=2)$. The delay spreads have not been evaluated at the black coloured points.

$\left|\left(S_{\tau}(r)-\hat{S}_{\tau}(r)\right) / S_{\tau}(r)\right|$, are demonstrated in Fig. 10 and Fig. 11, respectively. The curves in Fig. 8 correspond to the location where the relative delay spread error is below 0.06 , while at the location corresponding to Fig. 9 the relative error is equal to 0.1 . It should be mentioned that the delay spread relative absolute errors larger than $10 \%$ might not be acceptable for detailed analysis of system performance.

Note the tendency in Figs. 10 and 11 that the relative delay spread error is larger at the points located further away from the transmitters. In the following subsection, we present more details related to the spatial distribution of the approximation errors.

\section{E. Spatial Distribution of Absolute Approximation Errors}

Figs. 12 and 13 demonstrates the spatial distribution of the absolute power approximation errors for the considered propagation scenarios at the frequency $\nu=2.345 \mathrm{GHz}$ provided by the $N=2$ term approximations.

It can be seen from Figs. 12 and 13 that there exists a correlation between the predicted power $P(r, \nu)$ and the absolute approximation error. Indeed, the smaller the power $P(r, \nu)$, the larger is the absolute approximation error $\mid \hat{P}(r, \nu)[\mathrm{dBm}]-$ $P(r, \nu)[d B m] \mid$. This observation is supported by the bivariate histograms in Figs. 14 and 15, which illustrate the distributions of the observation points w.r.t. the predicted power $P(r, \nu)$ and the absolute power approximation error.

An interesting implication of the noted correlation between the absolute approximation error and the predicted power is a possibility of choosing the number of terms in (16) dependent on the local predicted power, e.g., the number of terms $N$ is larger for the regions located further away from a transmitter. This way the absolute approximation error can be lowered without significant increase in the computational complexity of the method.

\section{CONCLUSION}

The computational complexity of the traditional MRFDPF method is dominated by the preprocessing step

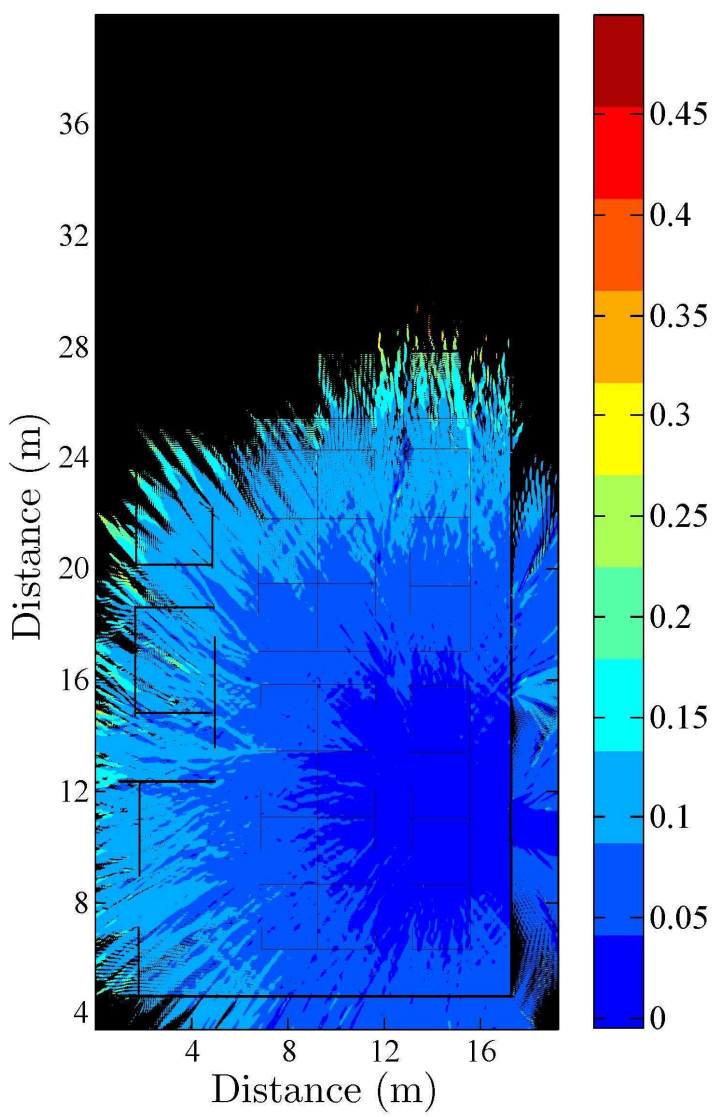

Fig. 11. Stanford. The delay spread relative absolute error $\mid\left(S_{\tau}(r)-\right.$ $\left.\hat{S}_{\tau}(r)\right) / S_{\tau}(r) \mid(N=2)$. The delay spreads have not been evaluated at the black coloured points.

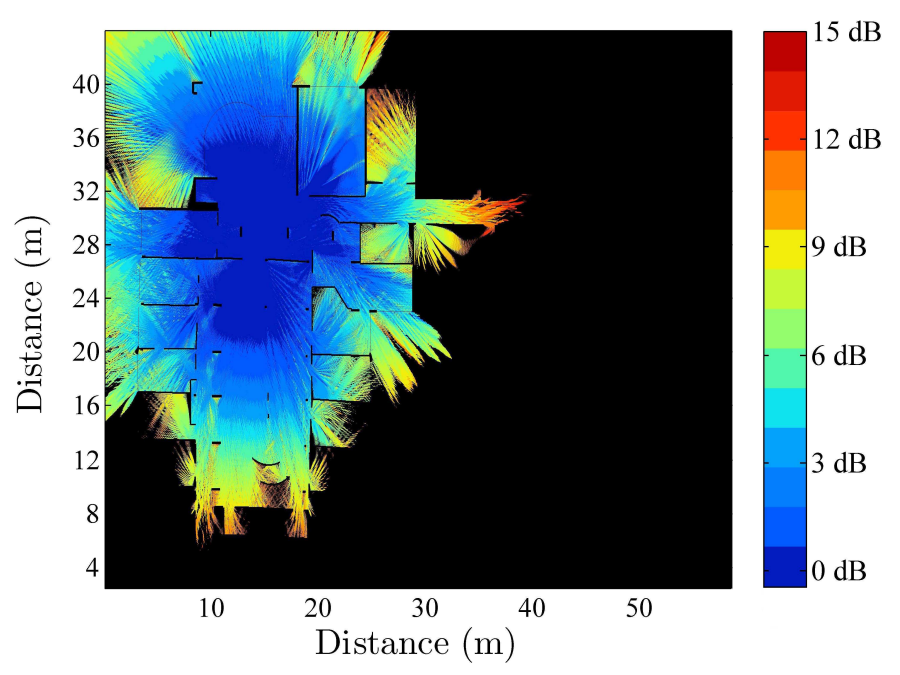

Fig. 12. CITI. The spatial distribution of the absolute power approximation error $|\hat{P}(r, \nu)[d B m]-P(r, \nu)[d B m]|$ at $\nu=2.345 \mathrm{GHz}$ and $N=2$ $\left(\nu_{0}=2.35 \mathrm{GHz}\right)$. The absolute power approximation error has not been evaluated at the block colored points.

especially for large indoor and outdoor propagation environments. In this paper, a new implementation of the MRFDPF method optimized for multifrequency simulations has been proposed. The new implementation reduces 


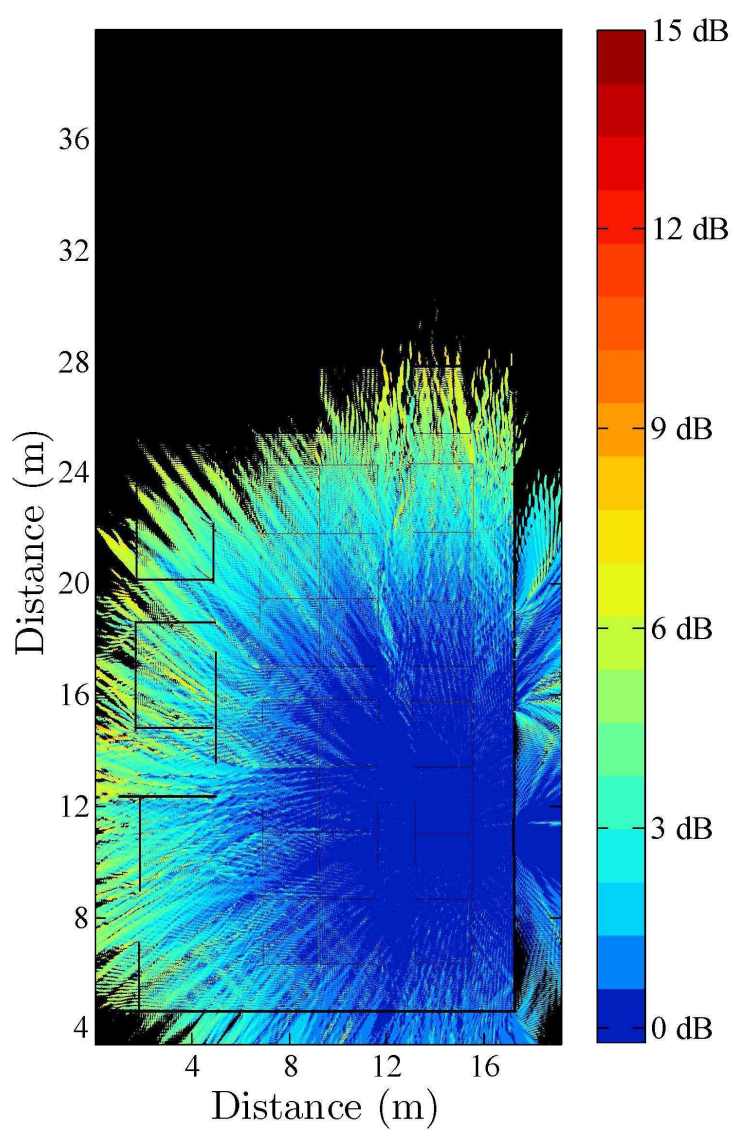

Fig. 13. Stanford. The spatial distribution of the absolute power approximation error $|\hat{P}(r, \nu)[d B m]-P(r, \nu)[d B m]|$ at $\nu=2.347 \mathrm{GHz}$ and $N=2$ $\left(\nu_{0}=2.35 \mathrm{GHz}\right)$. The absolute power approximation error has not been evaluated at the block colored points.

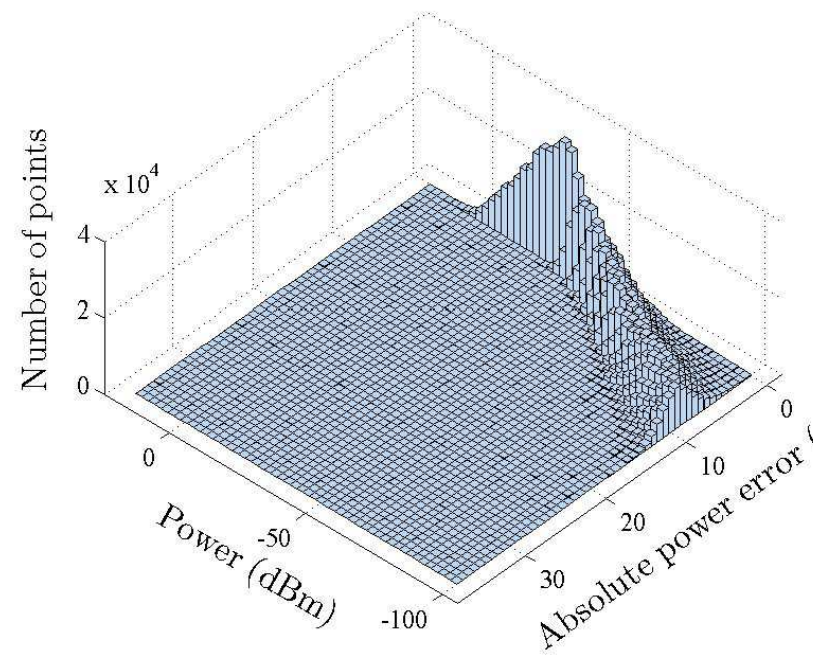

Fig. 14. CITI. The distribution of the observation points w.r.t. the predicted power and the absolute power approximation error $(\nu=2.347 \mathrm{GHz}, N=2$, $\nu_{0}=2.35 \mathrm{GHz}$ ).

the computational cost of the radio coverage prediction at multiple frequencies by conducting the preprocessing step only for one frequency. A linear reduction in the computational cost (considered as a function of the size

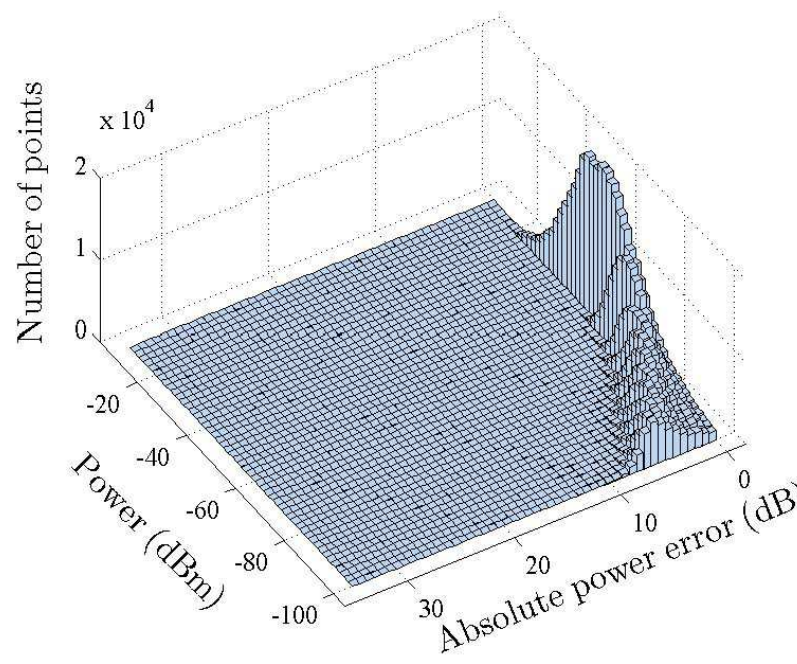

Fig. 15. Stanford. The distribution of the observation points w.r.t. the predicted power and the absolute power approximation error $(\nu=2.347 \mathrm{GHz}$, $N=2, \nu_{0}=2.35 \mathrm{GHz}$ ).

of an abstract square propagation environment) has been anticipated.

In addition to that, we have demonstrated that the MRFD approach can be applied to the well-known TLM method. Theoretical analysis of the proposed MR-FDTLM method optimized for multifrequency simulation predicts a quadratic reduction in the computational load when applied to model a 3D (cubical) propagation environment.

Unfortunately, the reduction in the computational complexity is achieved at the expanse of degradation in the accuracy of the radio coverage prediction. In general, the accuracy of the prediction or, alternatively, the frequency bandwidth, in which a specified accuracy is sustained, is dependent on the modeled propagation environment.

The experimental results provided in the paper include two examples of employing the proposed implementation of the MR-FDPF method for predicting the radio coverage in a range of frequencies. For both considered indoor propagation environments, an approximately $50 \%$ reduction in the computational cost has been achieved by using one-term approximation $(N=1)$ in (16). The resulting approximation can be characterized by the 1-dB error relative bandwidth, which is equal to $0.1 \%(0.2 \%)$ for the first (second) considered propagation environment. Also, the new implementation of the MR-FDPF method exhibits $5 \mathrm{~dB}(3 \mathrm{~dB})$ absolute power error at the edges of the $\% B=0.4 \%(10 \mathrm{MHz} / 2.35 \mathbf{G H z})$ relative error bandwidth. The two-terms $(N=2)$ approximation (16) reduces the computational load by $20 \%(30 \%)$, while the average 1 -dB error relative bandwidth becomes $0.25 \%(0.3 \%)$ and the absolute power error of $4 \mathrm{~dB}(2 \mathrm{~dB})$ occurs at the boarders of the $\% B=0.4 \%$ relative bandwidth. The computational complexity of the proposed and the traditional implementations of the MR-FDPF method become equal when $N=3$, which is sufficiently lower than the theoretically predicted value $N=11(N=6)$. Partly, this 
inconsistency can be explained by the roughness of the method used for assessing the computational complexity as well as by the lack of optimization of the actual software MR-FDPF method realization.

It has been noticed that the prediction accuracy degrades as the distance from the transmitter increases. This observation opens additional possibilities to improve the accuracy of the radio coverage prediction without significant increase in the computational complexity of the proposed MR-FDPF and MR-FDTLM methods.

\section{APPENDIX}

Similar to the scattering equation (24), which describes the relationship between the incident and reflected voltages for a single SSCN node, we can write the scattering equation for the block of nodes $A$

$$
\overrightarrow{\mathbf{V}}_{A}=\boldsymbol{\Sigma}_{A} \overleftarrow{\mathbf{V}}_{A}+\overrightarrow{\mathbf{S}}_{A}
$$

where the excitation signals aggregated in the vector $\overrightarrow{\mathbf{S}}_{A}$ correspond to all sources located inside the block $A$. Although omitted for simplifying the notation in (37), the dependence on the frequency $\nu$ and the location $r$ is understood.

Suppose the block $A$ and the block $B$ are connected by $k$ lines. Then, the scattering equations (37) can be written (perhaps, after a matrix/vector permutation) for the blocks $A$ and $B$ as follows

$$
\begin{aligned}
& {\left[\begin{array}{ll}
\boldsymbol{\Sigma}_{A_{11}} & \boldsymbol{\Sigma}_{A_{12}} \\
\boldsymbol{\Sigma}_{A_{21}} & \boldsymbol{\Sigma}_{A_{22}}
\end{array}\right]\left[\begin{array}{l}
\overleftarrow{\mathbf{V}}_{A_{1}} \\
\overleftarrow{\mathbf{V}}_{A_{2}}
\end{array}\right]+\left[\begin{array}{l}
\overrightarrow{\mathbf{S}}_{A_{1}} \\
\overrightarrow{\mathbf{S}}_{A_{2}}
\end{array}\right]=\left[\begin{array}{l}
\overrightarrow{\mathbf{V}}_{A_{1}} \\
\overrightarrow{\mathbf{V}}_{A_{2}}
\end{array}\right], \begin{array}{l}
\} N_{A}-k \\
\}
\end{array}} \\
& {\left[\begin{array}{ll}
\boldsymbol{\Sigma}_{B_{11}} & \boldsymbol{\Sigma}_{B_{12}} \\
\boldsymbol{\Sigma}_{B_{21}} & \boldsymbol{\Sigma}_{B_{22}}
\end{array}\right]\left[\begin{array}{c}
\overleftarrow{\mathbf{V}}_{B_{1}} \\
\overleftarrow{\mathbf{V}}_{B_{2}}
\end{array}\right]+\left[\begin{array}{l}
\overrightarrow{\mathbf{S}}_{B_{1}} \\
\overrightarrow{\mathbf{S}}_{B_{2}}
\end{array}\right]=\left[\begin{array}{l}
\overrightarrow{\mathbf{V}}_{B_{1}} \\
\overrightarrow{\mathbf{V}}_{B_{2}}
\end{array}\right], \begin{array}{l}
\} N_{B}-k \\
\} k
\end{array}}
\end{aligned}
$$

where the subvectors $\overleftarrow{\mathbf{V}}_{A_{2}}, \overrightarrow{\mathbf{V}}_{A_{2}}, \overleftarrow{\mathbf{V}}_{B_{2}}$, and $\overrightarrow{\mathbf{V}}_{B_{2}}$, which correspond to the $k$ lines connecting the blocks $A$ and $B$, are related as

$$
\overleftarrow{\mathbf{V}}_{A_{2}}=\overrightarrow{\mathbf{V}}_{B_{2}} \quad \overrightarrow{\mathbf{V}}_{A_{2}}=\overleftarrow{\mathbf{V}}_{B_{2}}
$$

Using (39), we obtained from (38)

$$
\begin{aligned}
& \boldsymbol{\Sigma}_{A_{22}} \overleftarrow{\mathbf{V}}_{A_{2}}-\overleftarrow{\mathbf{V}}_{B_{2}}=-\boldsymbol{\Sigma}_{A_{21}} \overleftarrow{\mathbf{V}}_{A_{1}}-\overrightarrow{\mathbf{S}}_{A_{2}} \\
& \overleftarrow{\mathbf{V}}_{A_{2}}-\boldsymbol{\Sigma}_{B_{22}} \overleftarrow{\mathbf{V}}_{B_{2}}=-\boldsymbol{\Sigma}_{B_{21}} \overleftarrow{\mathbf{V}}_{B_{1}}-\overrightarrow{\mathbf{S}}_{B_{2}}
\end{aligned}
$$

and consequently

$$
\begin{aligned}
\mathbf{V}_{A_{2}} & =\left(\mathbf{I}-\boldsymbol{\Sigma}_{B_{22}} \boldsymbol{\Sigma}_{A_{22}}\right)^{-1}\left(\boldsymbol{\Sigma}_{B_{21}} \overleftarrow{\mathbf{V}}_{B_{1}}+\boldsymbol{\Sigma}_{B_{22}} \boldsymbol{\Sigma}_{A_{21}} \overleftarrow{\mathbf{V}}_{A_{1}}\right) \\
& +\left(\mathbf{I}-\boldsymbol{\Sigma}_{B_{22}} \boldsymbol{\Sigma}_{A_{22}}\right)^{-1}\left(\overrightarrow{\mathbf{S}}_{B_{2}}+\boldsymbol{\Sigma}_{B_{22}} \overrightarrow{\mathbf{S}}_{A_{2}}\right) \\
\mathbf{V}_{B_{2}} & =\left(\mathbf{I}-\boldsymbol{\Sigma}_{A_{22}} \boldsymbol{\Sigma}_{B_{22}}\right)^{-1}\left(\boldsymbol{\Sigma}_{A_{21}} \overleftarrow{\mathbf{V}}_{A_{1}}+\boldsymbol{\Sigma}_{A_{22}} \boldsymbol{\Sigma}_{B_{21}} \overleftarrow{\mathbf{V}}_{B_{1}}\right) \\
& +\left(\mathbf{I}-\boldsymbol{\Sigma}_{A_{22}} \boldsymbol{\Sigma}_{B_{22}}\right)^{-1}\left(\overrightarrow{\mathbf{S}}_{A_{2}}+\boldsymbol{\Sigma}_{A_{22}} \overrightarrow{\mathbf{S}}_{B_{2}}\right) .
\end{aligned}
$$

The scattering equation for the combined block $A B$ can now be determined by excluding the subvectors $\overleftarrow{\mathrm{V}}_{A_{2}}, \overrightarrow{\mathrm{V}}_{A_{2}}$,
$\overleftarrow{\mathbf{V}}_{B_{2}}$, and $\overrightarrow{\mathbf{V}}_{B_{2}}$ from the consideration

$$
\left[\begin{array}{ll}
\boldsymbol{\Sigma}_{A B_{11}} & \boldsymbol{\Sigma}_{A B_{12}} \\
\boldsymbol{\Sigma}_{A B_{21}} & \boldsymbol{\Sigma}_{A B_{22}}
\end{array}\right]\left[\begin{array}{c}
\overleftarrow{\mathbf{V}}_{A_{1}} \\
\overleftarrow{\mathbf{V}}_{B_{1}}
\end{array}\right]+\overrightarrow{\mathbf{S}}_{A B}=\left[\begin{array}{l}
\overrightarrow{\mathbf{V}}_{A_{1}} \\
\overrightarrow{\mathbf{V}}_{B_{1}}
\end{array}\right], \begin{array}{ll}
\} & N_{A}-k \\
N_{B}-k
\end{array}
$$

where

$$
\begin{aligned}
& \boldsymbol{\Sigma}_{A B_{11}}=\boldsymbol{\Sigma}_{A_{11}}+\boldsymbol{\Sigma}_{A_{12}}\left(\mathbf{I}-\boldsymbol{\Sigma}_{B_{22}} \boldsymbol{\Sigma}_{A_{22}}\right)^{-1} \boldsymbol{\Sigma}_{B_{22}} \boldsymbol{\Sigma}_{A_{21}} \\
& \boldsymbol{\Sigma}_{A B_{12}}=\boldsymbol{\Sigma}_{A_{12}}\left(\mathbf{I}-\boldsymbol{\Sigma}_{B_{22}} \boldsymbol{\Sigma}_{A_{22}}\right)^{-1} \boldsymbol{\Sigma}_{B_{21}} \\
& \boldsymbol{\Sigma}_{A B_{21}}=\boldsymbol{\Sigma}_{B_{12}}\left(\mathbf{I}-\boldsymbol{\Sigma}_{A_{22}} \boldsymbol{\Sigma}_{B_{22}}\right)^{-1} \boldsymbol{\Sigma}_{A_{21}} \\
& \boldsymbol{\Sigma}_{A B_{22}}=\boldsymbol{\Sigma}_{B_{11}}+\boldsymbol{\Sigma}_{B_{12}}\left(\mathbf{I}-\boldsymbol{\Sigma}_{A_{22}} \boldsymbol{\Sigma}_{B_{22}}\right)^{-1} \boldsymbol{\Sigma}_{A_{22}} \boldsymbol{\Sigma}_{B_{21}}
\end{aligned}
$$

and

$$
\begin{aligned}
\overrightarrow{\mathbf{S}}_{A B} & =\left[\begin{array}{l}
\overrightarrow{\mathbf{S}}_{A_{1}} \\
\overrightarrow{\mathbf{S}}_{B_{1}}
\end{array}\right] \\
& +\left[\begin{array}{l}
\boldsymbol{\Sigma}_{A_{12}}\left(\mathbf{I}-\boldsymbol{\Sigma}_{B_{22}} \boldsymbol{\Sigma}_{A_{22}}\right)^{-1}\left(\overrightarrow{\mathbf{S}}_{B_{2}}+\boldsymbol{\Sigma}_{B_{22}} \overrightarrow{\mathbf{S}}_{A_{2}}\right) \\
\boldsymbol{\Sigma}_{B_{12}}\left(\mathbf{I}-\boldsymbol{\Sigma}_{A_{22}} \boldsymbol{\Sigma}_{B_{22}}\right)^{-1}\left(\overrightarrow{\mathbf{S}}_{A_{2}}+\boldsymbol{\Sigma}_{A_{22}} \overrightarrow{\mathbf{S}}_{B_{2}}\right)
\end{array}\right]
\end{aligned}
$$

\section{ACKNOWLEDGMENT}

The authors would like to thank Dr. Nicolai Czink from FTW, Vienna, Austria, for providing the data from the measurement campaign conducted in Stanford University.

This project is funded by the FP7 IPLAN Project.

\section{REFERENCES}

[1] U. S. Jha and R. Prasad, OFDM. Towards Fixed and Mobile Broadband Wireless Access. Artech House, 2007.

[2] J. Daniel G. Swanson and W. J. R. Hoefer, Microwave Circuit Modeling Using Electromagnetic Field Simulation. Artech House, 2003.

[3] R. Hoppe, P. Wertz, F. M. Landstorfer, and G. Wölfle, "Advanced rayoptical wave propagation modelling for urban and indoor scenarious including wideband properties," European Transactions on Telecoттиnications, vol. 14, pp. 61-69, 2003.

[4] A. F. Molisch, Wireless Communications, 2nd ed. Chichester: WileyIEEE Press, 2011

[5] J.-M. Gorce, K. Jaffrès Runser, and G. De La Roche, "The Adaptive Multi-Resolution Frequency-Domain ParFlow (MR-FDPF) Method for Indoor Radio Wave Propagation Simulation. Part I : Theory and Algorithms," INRIA, Tech. Rep. RR-5740, 11 2005. [Online]. Available: http://hal.inria.fr/inria-00070278/PDF/RR-5740.pdf

[6] P. Berini and K. Wu, "A new frequency domain symmetrical condensed tlm node," IEEE Microw. Guided Wave Lett., vol. 4, no. 6, pp. 180-182, 1994.

[7] D. Johns and C. Christopouros, "New frequency-domain tlm method for the numerical solution of steady-state electromagnetic problems," IEE Proceedings -Science, Measurement and Technology, vol. 141, no. 4, pp. 310-316, 1994.

[8] H. Jin and R. Vahldieck, "A new Frequency-Domain TLM symmetrical condensed node derived directly from Maxwell's equations," in Proc. IEEE MTT-S Int. Microwave Symp. Digest, 1995, pp. 487-490.

[9] B. Chopard, P. Luthi, and J.-F. Wagen, "Lattice-Boltzmann method for wave propagation in urban microcells," Microwaves, Antennas and Propagation, IEE Proceedings, vol. 144, no. 4, pp. 251 -255, aug 1997.

[10] C. Christopoulos, The Transmission-Line Modeling (TLM) Method in Electromagnetics. Morgan \& Claypool Publishers, 2006.

[11] I. Salama and S. M. Riad, "TFDTLM - a new computationally efficient frequency-domain transmission-line-matrix method," IEEE Trans. Microw. Theory Tech., vol. 48, no. 7, pp. 1089-1097, 2000.

[12] J.-M. Gorce, K. Jaffres-Runser, and G. de la Roche, "Deterministic approach for fast simulations of indoor radio wave propagation," IEEE Trans. Antennas Propag., vol. 55, no. 3, pp. 938-948, 2007. 
[13] G. de la Roche, K. Jaffres-Runser, and J.-M. Gorce, "On predicting in-building WiFi coverage with a fast discrete approach," International Journal of Mobile Network Design and Innovation, vol. 2, pp. 3-12, 2007.

[14] B. Chopard and M. Droz, Cellular Automata Modeling of Physical Systems. Canbridge University Press, 1998.

[15] P. O. Luthi, "Lattice wave automata: From radio waves to fractures propagation," Ph.D. dissertation, University of Geneva, 1998.

[16] B. Chopard and P. O. Luthi, "Lattice-Boltzmann computations and applications to physics," Theor. Comput. Sci., vol. 217, no. 1, pp. 115$130,1999$.

[17] C. D. Meyer, Matrix Analysis and Applied Linear Algebra. SIAM, 2001.

[18] V. Trenkic, "The development and characterization of advanced nodes for the TLM method," Ph.D. dissertation, University of Nottingham, 1995.

[19] P. B. Johns, "A symmetrical condensed node for the tlm method," IEEE Trans. Microw. Theory Tech., vol. 35, no. 4, pp. 370-377, 1987.

[20] P. Enders, "Huygens' principle in the transmission line matrix method (tlm). global theory," Int. J. Numer. Model., vol. 14, pp. 451-456.

[21] N. V. Kantartzis and T. D. Tsiboukis, Modern EMC Analysis Techniques. Morgan \& Claypool Publishers, 2008, vol. Volume I: Time-Domain Computational Schemes.

[22] V. V. Nikolsky and T. I. Nikolskaya, Electrodynamics and Propagation of Radio Waves. Moscow, Russia: Nauka, 1989, in Russian.

[23] G. D. Durgin, Space-Time Wireless Channels. NJ: Prentice Hall, 2003. 